\title{
RESTAURACIÓN DEL PAISAJE DE LA MONTAÑA ESPAÑOLA CON GANADERÍA: UN ENSAYO EN LA CORDILLERA CANTÁBRICA COMBINANDO PASTOREO DE CAPRINO Y PODA DE MATORRALES
}

\section{Landscape restoration due to goat grazing in the Spanish mountain. An test in the Cantabrian mountain, combining grazing and shrub pruning}

\author{
J. Álvarez Martínez ${ }^{1}$, M. P. Errea Abad ${ }^{2}$, A. Gómez-Villar ${ }^{3}$ y T. Lasanta ${ }^{2 *}$ \\ ${ }^{1}$ Departamento de Ingeniería Agrícola y Forestal. Universidad de Valladolid. Avda. Madrid, 44. 34004 - Palencia. gestion@iaf.uva.es \\ ${ }^{2}$ Instituto Pirenaico de Ecología (CSIC). Avda Montañana 1005. Apdo.13034.50059-Zaragoza.paz@ipe.csic.es y fm@ipe.csic.es \\ ${ }^{3}$ Departamento de Geografía y Geología. Universidad de León. Campus de Vegazana s/n. 24071 - León. agomv@unileon.es \\ *Autor corresponsal
}

Recibido: 10-11-2014. Aceptado: 27-02-2015. Fecha de publicación on-line: 10-09-2015

Citation / Cómo citar este artículo: Álvarez Martínez, J., Errea Abad, M. P., Gómez-Villar, A. \& Lasanta, T. (2015). Restauración del paisaje de la montaña española con ganadería: Un ensayo en la Cordillera Cantábrica combinando pastoreo de caprino y poda de matorrales. Pirineos, 170, e008. doi: http://dx.doi.org/10.3989/Pirineos.2015.170001

\begin{abstract}
RESUMEN: Se analiza qué papel puede desempeñar el pastoreo con cabras domésticas, en combinación con la poda de Cytisus scoparius, en el control de la expansión de los matorrales y, especialmente, en la configuración y fragmentación del paisaje. Se trabaja con dos cargas ganaderas: simple (4,5 cabras/ha/año) y doble ( 9 cabras/ha/año). Los resultados ponen de relieve que la poda por sí sola tiene escasa capacidad para limitar la expansión de los matorrales. La combinación de poda y carga de ganado simple retrasa el avance de los matorrales. Más eficaz resulta la carga doble junto a la poda, ya que permite controlar la expansión en superficie de Cytisus scoparius y también su altura. Se comprueba, también, que la carga doble mantiene un paisaje más fragmentado que la carga simple, dando lugar a una estructura de pequeñas manchas, lo que resulta muy interesante tanto desde una perspectiva ganadera como ambiental.
\end{abstract}

PALABRAS CLAVE: Pastoreo; cabras; matorrales; paisaje cultural; montaña; España.

\begin{abstract}
Depopulation in Spanish mountain areas and livestock decrease, which traditionally grazed pasture lands, have favored an uncontrolled expansion of shrubs and, consequently, a significant increase of the fuel material and the fire risk, and the decrease of the landscape diversity. In the Cantabrian Mountain, Cytisus scoparius shows a large capacity to colonize abandoned lands and at present it is covering large areas, which were grazed before the abandonment.

This paper analyzes the role of goats grazing, in combination with Cytisus scoparius pruning, to control the shrub spread, and how these actions are affecting the landscape configuration and fragmentation. The analysis was based on experimental plots, which combined two stocking rates: single ( 4.5 goats/ha/year) and double ( 9 goats/ha/year). The results show a small capacity of the sole application of pruning to limit the shrub expansion, since three years after the plants recovered the $90 \%$ of the initial coverage. The combination of pruning and single stocking rate delays
\end{abstract}




\section{2 •J. ÁLVAREZ MARTÍNEZ, M. P. ERREA ABAD, A. GÓMEZ-VILLAR, T. LASANTA}

the advance of shrubs, but in four years recover the $70.6 \%$ of its height and the $64 \%$ of its length. More effective is the double rate, as it allows controlling the expansion of Cytisus scoparius, since four years after the experiment the shrub branches only grew $10 \mathrm{~cm}$ in an average.

Double stocking rate also preserves a more fragmented landscape than the single rate; it produces a land structure characterized by small patches, which is more interesting from an environmental point of view.

KEYWORDS: Grazing; goats; shrubs; cultural landscape; mountain; Spain.

\section{Introducción}

Desde mediados del siglo xx las montañas españolas sufren un proceso de marginación económica, con pérdida de más del $60 \%$ de sus habitantes, abandono masivo del espacio agrícola y disminución de la ganadería extensiva, con escasa utilización de los pastos naturales (Lasanta, 1990; Collantes, 2004). En el territorio dicho proceso se manifiesta, sobre todo, en la revegetación de amplios sectores con el avance de matorrales y bosques de sucesión (Bielsa et al., 2005; Nogués-Bravo, 2006; Lasanta \& Vicente-Serrano, 2007; Pueyo \& Beguería, 2007; Vila Subirós et al., 2009, entre otros).

La recuperación de la cubierta vegetal tiene algunas ventajas medioambientales y económicas como la mayor absorción de $\mathrm{CO}_{2}$ (Robert \& Saugier, 2003), la disminución de la erosión del suelo en las laderas (García-Ruiz \& López Bermúdez, 2009), la mejora de la calidad de las aguas de escorrentía y la disminución del ritmo de aterramiento de embalses (López-Moreno et al., 2003), y el incremento de la producción de madera (Ortuño \& MartínFernández, 2006). Pero también tiene algunos efectos negativos como el incremento del material combustible y el riesgo de incendios (Koutsias et al., 2010), la menor disponibilidad de agua en las cuencas (García-Ruiz et al., 2010), la disminución de la diversidad y del valor estético del paisaje (Lasanta et al., 2005; Rescia et al., 2008; Sayadi et al., 2009), la pérdida de recursos pastorales (Molinillo et al., 1997; Arnáez et al.,2011), y la degradación de la flora y la fauna asociadas a paisajes culturales, como los de las montañas españolas (Suárez-Seoane et al., 2002). Entre los efectos negativos del proceso de revegetación tiene especial importancia en nuestras montañas, y en general en las de los países de dilatada historia, la pérdida de paisajes humanizados. En este sentido, Farina (2007) argumenta que el paisaje de referencia en las regiones euro-mediterráneas es un paisaje cultural originado por la ganadería extensiva.

Con el fin de minimizar los impactos negativos del avance de los matorrales, numerosos estudios científicos reclaman la intervención de la Administración en el territorio. Plantean el uso de la ganadería extensiva como herramienta de control para detener la progresión de los matorrales (Bernués et al., 2007; Ruiz Mirazo et al., 2011; Bartolomé et al., 2011). Sin embargo, a pesar del interés del tema, no son muy numerosos los trabajos que cuantifican el efecto real del ganado sobre la vegetación y su dinámica. Valderrábano y Torrano (2000) comprobaron que la eficacia de la cabra en el control de Genista scorpius era mayor si el pastoreo se realizaba en otoño y con cargas elevadas. Riedel (2007) realizó un experimento en pastos arbustivos de la Sierra de Guara (Pirineo aragonés) con pastoreo de ovino (cargas medias de 0,15 UGM/ha/ año), comprobando que el pastoreo supuso una reducción de la dinámica de la vegetación, pero no logró detenerla; también comprobó que el efecto difería mucho entre especies. Casasús et al. (2007) utilizaron una carga de 2 UGM/ha/año de vacas nodrizas en pastos ralos del valle de la Garcipollera (Pirineo aragonés). Encontraron un efecto claramente significativo del pastoreo del ganado, que fue capaz de mantener estable la biomasa arbustiva. Sin embargo, en las áreas no pastadas observaron una rápida e intensa densificación de la vegetación arbustiva. Otros autores muestran cómo la ausencia del pastoreo conduce a una rápida expansión de los matorrales en diferentes montañas europeas (Gellrich et al., 2007; Olsson et al., 2000; Tasser et al., 2007; Mottet et al., 2006).

Los estudios realizados llegan, pues, a la conclusión de que la ganadería extensiva, por sí sola, es incapaz de controlar el proceso de revegetación, por lo que se deben ensayar prácticas que combinen el pastoreo del ganado con otras actuaciones. Existe ya información sobre los efectos del pastoreo y el desbroce mediante maquinaria (Lasanta et al., 2009 y 2015; Ruiz Mirazo, 2011) y con el uso de pequeños fuegos controlados (Gómez-García et al., 2011). Pero no hay estudios sobre el binomio ganadería extensiva - poda del matorral.

En este trabajo se analiza qué papel puede desempeñar el pastoreo con cabras en combinación con la poda en el control de los matorrales y en la estructura del paisaje, como síntesis del valor ambiental de un territorio. Se utiliza la cabra porque, del ganado doméstico presente en las montañas españolas, es el animal mejor adaptado al consumo de vegetación arbustiva altamente lignificada y defendida por compuestos químicos potencialmente tóxicos y defensas físicas (Hofmann, 1989). Además, se conoce bien su capacidad como "herramienta desbrozadora" de matorrales (Trías, 1996; Hart, 2001; Torrano \& Valderrábano, 2005; Glasser et al., 2012).

\section{Material y métodos}

\section{1. Área de estudio}

La zona de estudio se localiza en el Norte de la provincia de León, comprendiendo la parte media del valle del río Torío, en la Cordillera Cantábrica (Figura 1). Se trata de un valle que discurre de Norte a Sur, con altitudes comprendidas entre $2189 \mathrm{~m}$ (Pico de Braña) y los $900 \mathrm{~m}$. 


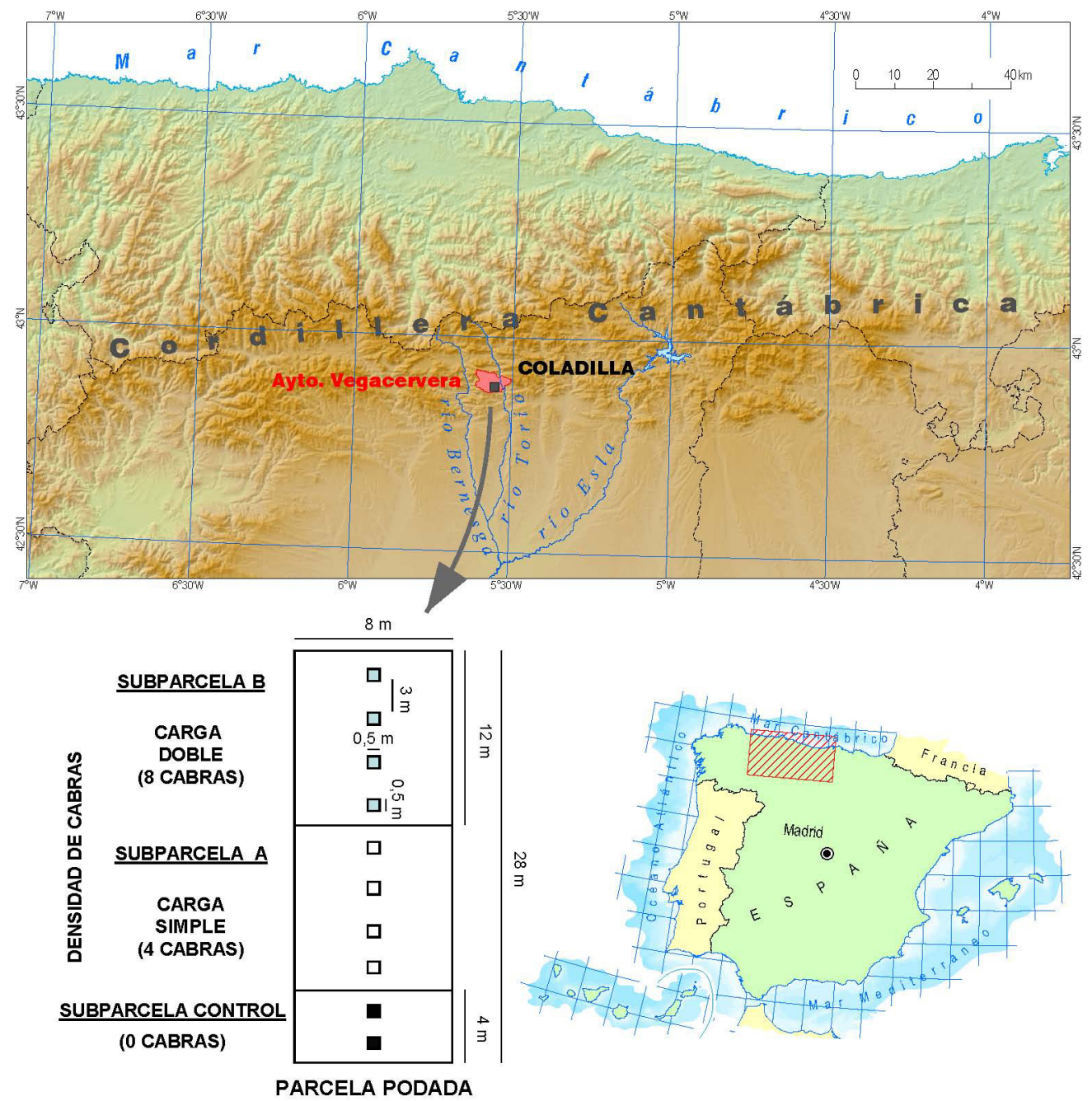

Figura 1: Área de estudio y esquema del dispositivo experimental.

Figure 1: The study area and experimental device.

La parte experimental de este estudio se llevó a cabo en el municipio de Vegacervera (34,9 $\mathrm{Km}^{2}$ de extensión y 1042 $\mathrm{m}$ de altitud), como se explicará más adelante.

La litología dominante corresponde a dolomías, calizas y calizas margosas. Los suelos muestran un $\mathrm{pH}$ ligeramente alcalino, con bajo contenido en materia orgánica y nitrógeno. La proporción de fósforo es muy variable, mientras que son suelos con altos contenidos de potasio, calcio y magnesio (Álvarez-Martínez, 1995). El clima es de tipo "Mediterráneo templado fresco" (Papadakis, 1966). La temperatura media anual en Vegacervera es de $9,8^{\circ} \mathrm{C}$ y la precipitación anual de $750 \mathrm{~mm}$, con un máximo invernal, siendo los registros más bajos en verano. El período de heladas seguras dura tres meses (desde mediados de noviembre a mediados de febrero). El periodo seco dura unos dos meses, coincidentes con el inicio del verano. En otoño es cuando se registra el máximo pluviométrico.
La vegetación potencial corresponde a Quercus pyrenaica o roble melojo (Linario trionithophorae-Querceto pyrenaicae sigmetum) (Rivas Martínez et al, 1987). En esta serie el óptimo sucesional o clímax es un bosque denso de talla mediana, en el que suele dominar el melojo (Quercus pyrenaica). Las etapas de regresión, al destruirse el bosque, comienzan por los piornales del Gestiniun polygaliphyllae, donde son comunes: Genista florida, Cytisus scoparius, Adenocarpus complicatus, Erica arborea y Pteridium aquilinum. Los brezales representan la etapa extrema de regresión y, por tanto, la menos productiva. En la actualidad están muy extendidos por el uso del fuego para regenerar pastos en las pasadas décadas.

En 1900 la población de Vegacervera alcanzaba los 940 habitantes, que se redujeron a 737 habitantes en 1960 (pérdida de 3,4 habitantes/año). Sin embargo, entre 1960 y 1970 el censo perdió 331 habitantes (33,1 habitantes/ año). En las décadas siguientes el municipio siguió per- 
diendo efectivos demográficos hasta registrar un mínimo en 1991 (287 habitantes); desde esta fecha el censo muestra un ligero repunte (347 habitantes en 2011). La pérdida de población implicó el abandono de la mayor parte del espacio agrícola y la caída de los censos ganaderos, especialmente en los años sesenta y setenta del pasado siglo. Según el Censo Agrario, en 1982 sólo había 320 UGM (0,09 UGM/ha) de ganado extensivo, que se incrementó en 1999 hasta 506 UGM, gracias a las subvenciones de la Política Agraria Comunitaria, para descender después a 352 UGM en 2009, por el excesivo envejecimiento de la población (Cascos Maraña, 2011). La baja presión ganadera soportada por el territorio desde mediados del siglo $\mathrm{XX}$ ha hecho que buena parte de las laderas se cubran de matorrales de sucesión, dominando Cytisus scoparius.

\subsection{Muestreo de campo}

La parte experimental se desarrolló en la localidad de Coladilla (municipio de Vegacervera), en una ladera agrícola dejada de cultivar en los años sesenta y aprovechada posteriormente con ganadería extensiva hasta dos décadas antes de iniciarse la experimentación. Se trata de una ladera representativa del sector meridional de la Cordillera Cantábrica, donde dominan los campos abandonados cubiertos ahora por Cytisus scoparius, como especie principal; unas matas se unen con otras dando la sensación de una cubierta muy densa que tapiza por completo la superficie. Otra característica es su elevado porte, con alturas medias de las matas superiores a $170 \mathrm{~cm}$, lo cual da lugar a la formación de varios estratos, con Rubus idaeus, Rosa sp. y Quercus pyrenaica en un estrato inferior a Cytisus scoparius. Para seleccionar el área experimental también se tuvo en cuenta el hecho de que había un ganadero dispuesto a colaborar en el estudio, mediante el pastoreo de su ganado en el momento que se le solicitaba.

Se delimitó y podó una parcela de matorral de $224 \mathrm{~m}^{2}$ ( $28 \times 8 \mathrm{~m})$ (ver Figura 1 y Foto 1$)$. La poda consistió en cortar con una hoz la cubierta arbustiva superior a una altura de $70 \mathrm{~cm}$ desde el suelo y la posterior retirada de la biomasa. Para analizar el efecto de dos intensidades de pastoreo se subdividió la parcela en dos subparcelas (Figura 1) con una superficie de $96 \mathrm{~m}^{2}(12 \times 8)$, que soportaron dos presiones de carga; y una tercera subparcela de $32 \mathrm{~m}^{2}$ (4 x 8), que sirvió de control al no recibir el impacto del pastoreo. Cada una de las 3 subparcelas se cercó con una estructura de malla de hierro galvanizado de dos metros de altura. Las intensidades de pastoreo fueron 4 cabras por subparcela (equivalente a 4,5 cabras/ha/año) y 8 cabras por subparcela (equivalente a 9 cabras/ha/año), que son los dos valores extremos utilizados en el área de estudio cuando se realiza pastoreo libre. La experimentación se mantuvo durante 4 años.

Dentro de la parcela se delimitaron 10 cuadrados $(50 \mathrm{~cm} \times 50 \mathrm{~cm})$ de muestreo, distribuidos 4 en cada subparcela pastoreada y 2 en la parcela control (Figura 1). Estos puntos, una vez ubicados, se fijaron y señalaron en el suelo con clavos gruesos, para que los muestreos en

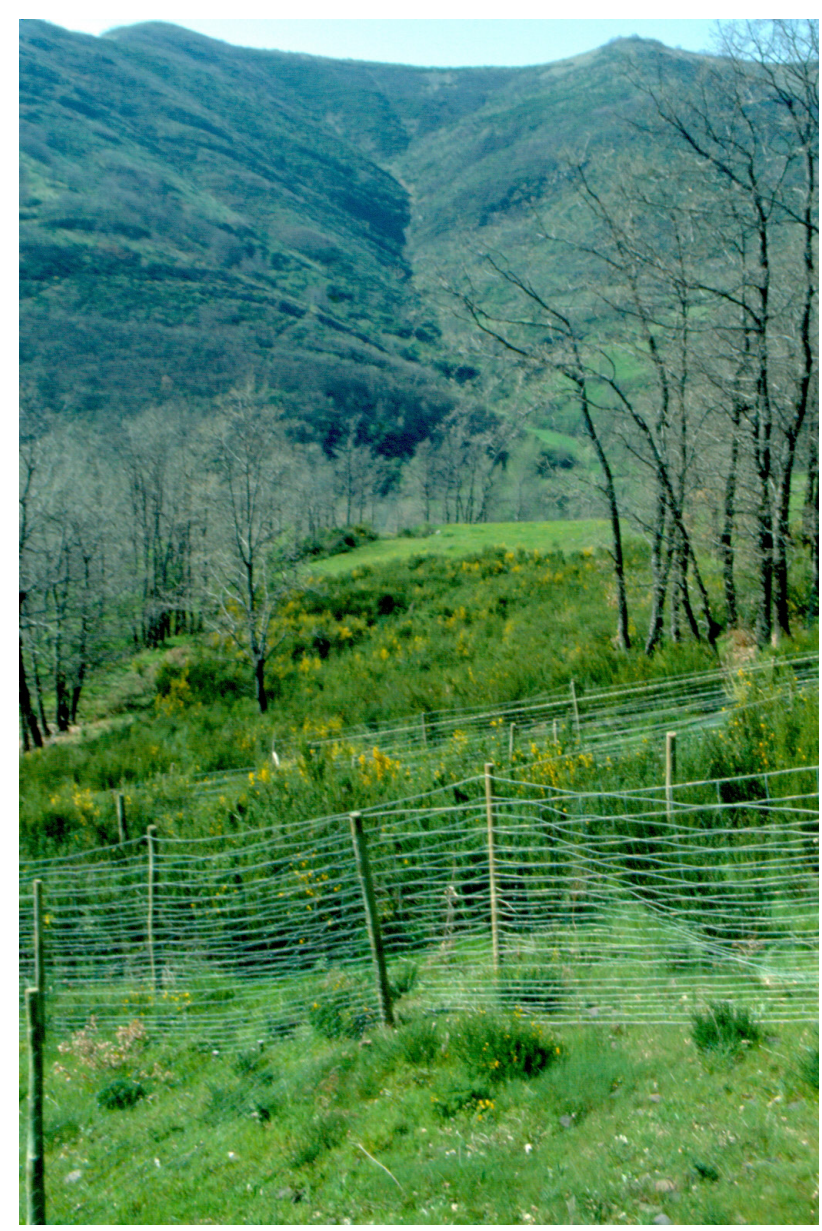

Foto 1: Perspectiva general del área de estudio y localización de la zona experimental.

Photo 1: Study area overview and location of the experimental area.

años posteriores coincidiesen siempre en el mismo lugar. En cada cuadrado se estimó, antes y después de cada pastoreo, la abundancia de especies herbáceas, el porcentaje de suelo cubierto por cada una de las especies del estrato arbustivo y por el conjunto de matorrales, la altura máxima de cada matorral, y los porcentajes de suelo desnudo, piedras, hojarasca, excrementos y otros restos orgánicos sueltos.

El pastoreo de las cabras (Foto 2) se realizó en dos momentos del año: primavera, coincidiendo con el inicio de la floración de Cytisus scoparius (principios de Junio), y en verano, en el inicio de la caída de las hojas y final de fructificación de dicho matorral (finales de Julio). En total, pues, se introdujo el ganado en las subparcelas 8 veces, con sus respectivos muestreos (ver Figura 2). Las cabras permanecían en cada subparcela dos días consecutivos para alcanzar cargas similares a las del área de estudio.

Con el fin de conocer el consumo de material arbustivo por parte del ganado, antes y después de cada pastoreo, se contó el número de matas, el número de ramas por 


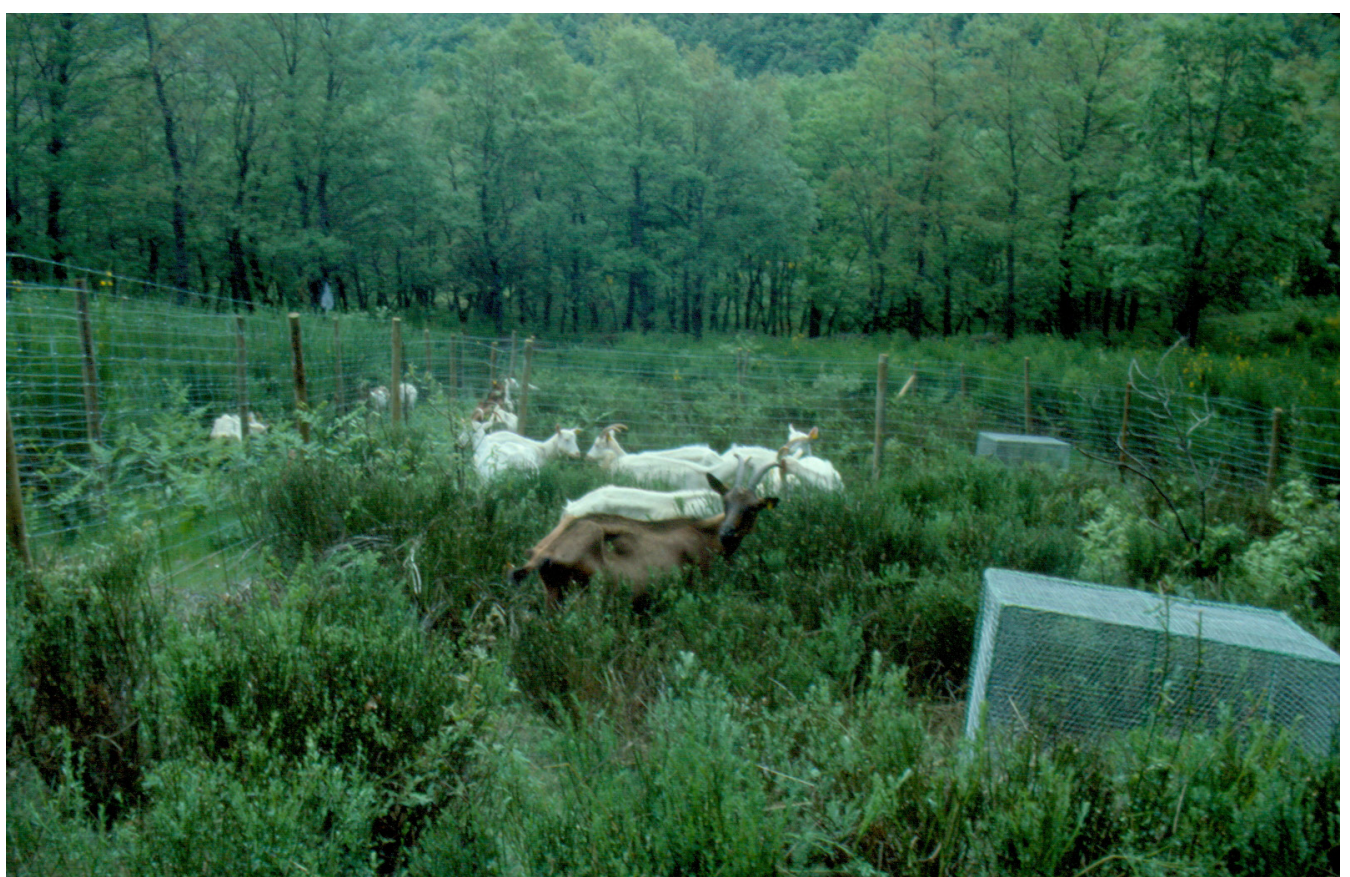

Foto 2: Pastoreo por cabras. En primer término con carga doble. Detrás con carga simple.

Photo 2: Goats grazing. In the first term including a double grazing rate; in the second term the areas with simple grazing rate are showed. Exclusion plots to calculate the grass production are also observed.

mata, el número de brotes del año por rama y se cartografió la distribución de las matas dentro de las subparcelas pastoreadas. Se calculó el volumen de cada mata y se midió la altura de la mata y de sus ramas. El volumen de las matas se calculó mediante el ajuste a un paraboloide de revolución, a partir de las medidas tomadas directamente de las plantas: altura máxima, diámetro mayor (distancia máxima en dirección longitudinal o transversal) y su perpendicular.La superficie del suelo cubierta por la planta se calculó mediante la fórmula del área de la elipse (Álvarez Martínez, 1995). Además, se contó el número de brotes del año y se calculó su peso mediante la recogida de doce brotes del año, que se introdujeron en bolsas de plástico para su traslado a laboratorio, donde se efectuó su pesado en verde. La materia seca (MS) se determinó mediante secado en estufa de aire forzado a $60^{\circ} \mathrm{C}$ durante 48 horas, tiempo suficiente para que los brotes del año pierdan la humedad sin que se produzcan cambios en su composición. Esta tarea se realizó antes y después del pastoreo. Es necesario señalar que se consideró consumo a la diferencia entre los brotes del año antes y después del pastoreo. Nos referimos con "porcentaje de consumo" a la proporción de brotes del año que han sido consumidos tras el pastoreo. Este porcentaje indica la preferencia por el recurso y el grado de "agotamiento" o "consumo" del mismo.

Para calcular la producción y el consumo de herbáceas en cada subparcela se segó la hierba de dos cuadrados de $30 \times 30 \mathrm{~cm}$ antes y después de cada pastoreo. El material herbáceo segado se introdujo en bolsas de plásti- co y se trasladó al laboratorio donde se efectuó su pesado en verde. La MS herbácea se determinó siguiendo el mismo procedimiento que para los brotes del año. Tanto los datos de producción arbustiva y herbácea como de consumo se expresan en $\mathrm{g} / \mathrm{m}^{2}$ de MS.

\subsection{Análisis del paisaje}

Para analizar el papel de la poda y el pastoreo en el paisaje se utilizaron métricas espaciales o índices de ecología del paisaje. Se trata de metodologías habituales en estudios de paisaje por aportar información sobre su configuración, incluyendo índices de organización espacial, fragmentación y dimensión fractal (Forman y Godron, 1986; Weng, 2007). La organización espacial y la fragmentación del paisaje se conoce por el número de parches o manchas, la superficie que ocupan, el tamaño medio de dichas manchas y su desviación estándar. Se aporta información también sobre los bordes de las manchas (longitud de ejes y tamaño medio). Además se analiza la dimensión fractal $(\mathrm{F})$, que permite conocer la complejidad del paisaje en relación con la forma del perímetro del conjunto de sus manchas (Mandelbrot, 1983; Kienast, 1993; McGarigal et al., 2002). Para llevar a cabo este análisis se ha utilizado el método perímetro-área, según el cual la dimensión fractal se calcula mediante la regresión de $\ln (\mathrm{A})$ frente a ln $(\mathrm{P} / 4)$, para cada uno de los tipos de paisaje incluidos en la unidad de análisis. La dimensión fractal se relaciona con la pendiente de la ecuación de ajuste de la 
regresión (m), según Lovejoy (1982), $\mathrm{F}=2 \mathrm{~m}$. Los resultados dan valores entre 1 (polígono totalmente regular) y 2. Se calcula también el índice medio de formas (IMF), que aporta información sobre la forma de la mancha, dando valores entre 1 (mancha totalmente circular) y 3 (mancha en forma de línea recta).

Para el cálculo de tales índices se empleó el programa V-LATE (Vector-Based Landscape Analysis Tools; extensión de ArcGis 10.0). Una síntesis de la formulación aplicada para el cálculo de los distintos parámetros y para la interpretación de los valores obtenidos puede consultarse, por ejemplo, en Errea et al. (2007).

Los estudios de la estructura del paisaje suelen realizarse a distintas escalas espaciales. Cuando se quiere obtener información a gran escala o de grano fino se selecciona una parte del territorio, lo que en la terminología se conoce como "ventana paisajística" (Burel y Baudry, 2002). Este análisis de una pequeña proporción del territorio permite obtener una información muy válida para cuantificar la heterogeneidad interna de un paisaje y, por lo tanto, para tener una idea aproximada de los rasgos paisajísticos más importantes de territorios más amplios. Dado que este estudio se basa en muestreos de parcelas de campo se consideró que cada una de las dos subparcelas pastadas constituye una ventana paisajística. Para ello se elaboró una cartografía detallada de cada subparcela pastada, posicionando y dimensionando las matas de Cytisus scoparius, antes y después de cada pastoreo. No se realizó la cartografía de la subparcela control porque al tercer año la vegetación arbustiva cubre ya el 100\% (ver Tabla 1), lo que significa que una sola mancha ocupa la totalidad de la subparcela. Suárez-Seoane (1998) aplicó la misma técnica, con resultados muy positivos, para estudiar los efectos ecológicos del abandono de tierras en la montaña de León.

\section{Resultados}

\subsection{Los efectos de la poda y el pastoreo sobre Cytisus scoparius}

La comunidad vegetal arbustiva presente en la parcela experimental al iniciarse el estudio tenía como especie dominante Cytisus scoparius (L.) Link con un $80 \%$ de cobertura, a la que acompañaban Rubus idaeus L. (60\%),
Rosa sp. (4\%) y algunos pies dispersos (3\%) de roble (Quercus pyrenaica Wild).

La edad de la población de Cytisus scoparius era de 10 años cuando se inició el experimento, información obtenida del propietario de la parcela. Uno de los rasgos fundamentales de la sucesión vegetal de Cytisus scoparius es que tiene un crecimiento rápido y coloniza pronto los campos abandonados. En nuestro caso la comunidad todavía no había alcanzado su máximo desarrollo, si bien presentaba rasgos de cierta madurez. De ahí, su alta cobertura $(80 \%)$, uniéndose unas matas con otras y dando la sensación de una cubierta muy densa que tapizaba por completo los campos. Otra característica era su elevado porte, con alturas medias superiores a los $170 \mathrm{~cm}$, lo que daba lugar a la formación de varios estratos, en el que el resto de componentes (Quercus pyrenaica, Rubus idaeus y Rosa sp.) se distribuían por debajo de Cytisus.

La poda de las ramas de altura superior a $70 \mathrm{~cm}$ implicó la disminución del 30\% aproximadamente de la cubierta de Cytisus scoparius; se extrajo $680 \mathrm{~g} / \mathrm{m}^{2}$ de biomasa, lo que representaba el $46 \%$ de la biomasa previa.

El cambio temporal en la subparcela control durante los cuatro años de experimentación puede seguirse en la Tabla 1. La poda acarrea una disminución inicial de la cubierta arbustiva a favor de la herbácea, pero rápidamente la primera se recupera. Así, la vegetación herbácea pasó del $15,8 \%$ el primer año al 56,3\% el segundo año, para disminuir los dos años siguientes hasta el 32,8\%. La vegetación arbustiva se redujo hasta el 79\% el segundo año, recuperándose durante los siguientes años. La cubierta de Cytisus scoparius alcanzó el 56\% durante el primer año y fue cubriendo progresivamente más superficie, de manera que al cuarto año ya ocupaba el 77,5\%, extensión muy próxima a la previa a la poda, si bien la altura de las plantas era inferior a la original. Rubus idaeus también se vio influida por la poda, pasando de un cubrimiento del 19\% el primer año al 39,3\% el cuarto año. Llama la atención la elevada presencia de suelo desnudo debajo de una cubierta densa de matorrales, con porcentajes que oscilan entre el $12,5 \%$ y el $20,5 \%$. La menor presencia de piedras con el paso del tiempo se explica porque las oculta el mayor cubrimiento de vegetación herbácea o arbustiva.

Durante la experimentación se comprobó que no germinaron semillas de Cytisus scoparius, debido, posiblemente, a que la reducción de la cubierta de matorral no fue suficiente para generar claros amplios que favorecie-

Tabla 1: Evolución de las medias de cubierta (\%) de las variables consideradas en la parcela control durante los cuatro años de observación. Table 1: Evolution of mean vegetation coverage (\%) of the variables considered in the control plot during the four years of observation.

\begin{tabular}{|c|c|c|c|c|c|c|c|c|}
\hline Años & Herbácea & Arbustiva & Cytisus & Rubus & Quercus & Rosa & $\begin{array}{c}\text { Suelo } \\
\text { Desnudo }\end{array}$ & Piedras \\
\hline $1 .^{\circ}$ & 15,8 & 79,3 & 56 & 19 & 4,3 & 0 & 12,5 & 2,5 \\
\hline $2 .^{\circ}$ & 56,3 & 79,0 & 51,3 & 27,8 & 0 & 0 & 18,8 & 1,3 \\
\hline $3 .^{\circ}$ & 41,8 & 100 & 62,5 & 37,5 & 0 & 0 & 15 & 0 \\
\hline $4 .^{\circ}$ & 32,8 & 116,8 & 77,5 & 39,3 & 0 & 0 & 20,5 & 0 \\
\hline
\end{tabular}


sen el incremento de la radiación lumínica sobre el suelo; las sombras generadas por la densa cubierta del matorral limitan el incremento de la temperatura del suelo, siendo la luz y la temperatura factores esenciales en la germinación de especies arbustivas (Puentes Graña, 1984).

En la subparcela pastoreada con carga doble, la producción media de brotes de primavera y verano fue de $81,7 \mathrm{~g} / \mathrm{m}^{2}$ de MS (Ver Tabla 2). El 66,9\% de los brotes los consumieron las cabras $\left(54,6 \mathrm{~g} / \mathrm{m}^{2}\right.$ de MS). Durante el verano el consumo de brotes fue ligeramente superior al de primavera $(70,8 \%$ frente al $63,1 \%)$, debido a que la hierba se agosta y es menos apetitosa, por lo que la cabra busca brotes tiernos en las especies arbustivas.

La subparcela gestionada con carga simple produjo una cantidad similar de brotes durante el primer año a la que soportó carga doble. Pero a partir del segundo año el incremento fue espectacular, llegándose a alcanzar los $280 \mathrm{~g} / \mathrm{m}^{2}$ de MS durante el segundo año, para estabilizarse a continuación en torno a los $155 \mathrm{~g} / \mathrm{m}^{2}$ de MS. Ello se explica porque el control de las cabras sobre Cytisus scoparius es insuficiente por la elevada oferta alimentaria, lo que hace que la alimentación del rumiante se complete con otros recursos (hierba, Rubus, Quercus). El consumo medio de brotes del año (Ver Tabla 2) representa el $40 \%$ de lo ofertado (113,2 $\mathrm{g} / \mathrm{m}^{2}$ de MS), que resulta ser más del doble de lo consumido en la subparcela con carga doble (54,6 g/ $\mathrm{m}^{2}$ de MS). El consumo es ligeramente superior en primavera $(43,8 \%)$ que en verano $(36,2 \%)$. La elevada cantidad y diversidad de recursos pastorales permite que el ungulado pueda seleccionar su dieta, tomando lo más apetecible en cada momento. De hecho, se comprobó que las matas de Rubus idaeus quedaron reducidas a la mitad con carga doble, mientras que apenas mostraron variación con carga simple.

La altura de la planta y su volumen son considerados como manifestaciones de la actividad fisiológica del desarrollo que presenta dicha planta; dan idea del poder de competencia por la luz frente a otras especies y de los cambios en su estructura por la acción de los herbívoros. En la subparcela control a los 4 años de la poda, las matas de Cytisus scoparius habían recuperado el $85 \%$ de su altura original, mientras que en la de carga simple la altura media de las ramas llegó a $120 \mathrm{~cm}(70,6 \%)$ y en la de carga doble sólo a $80 \mathrm{~cm}(47 \%)$. Respecto al volumen se comprobó que la carga de 9 cabras/ha/año redujo el doble el volumen medio de las matas que la carga simple, con valores de $1 \mathrm{~m}^{3}$ y $0,5 \mathrm{~m}^{3}$, respectivamente.

La poda implica crear espacios para el desarrollo de la vegetación herbácea. Los valores medios de producción herbácea fueron en primavera de $132,6 \mathrm{~g} / \mathrm{m}^{2}$ de MS con carga simple y de $160 \mathrm{~g} / \mathrm{m}^{2}$ de MS con carga doble (Tabla 2). En verano las producciones fueron sensiblemente más bajas $\left(94,4\right.$ y $88,5 \mathrm{~g} / \mathrm{m}^{2}$ de MS, respectivamente). La evolución a lo largo de los 8 muestreos efectuados en los 4 años de experimentación se refleja en la Figura 2. En la subparcela podada con carga simple todos los valores son inferiores a $150 \mathrm{~g} / \mathrm{m}^{2}$ de MS, mientras que con carga doble se sitúa por encima la producción de primavera del cuarto año. En la Figura 2 también se observa que con carga simple hay una tendencia a la disminución de la biomasa herbácea, mientras que en la parcela con carga doble se produce la evolución contraria, sobre todo en primavera. Ello se explica por el efecto inverso que producen ambas cargas: mientras la simple es incapaz de detener la recuperación del matorral, la doble permite mantener espacios abiertos en los que crece el pasto. Otro aspecto a destacar es que durante los dos primeros años las herbáceas se recuperan entre el pastoreo de primavera y verano, cosa que no ocurre durante el tercer y cuarto año, posiblemente por coincidir con años más secos.

De la producción herbácea las cabras consumieron (valores medios) 48,5 g/ $\mathrm{m}^{2} /$ año de $\mathrm{MS}$ en primavera y $21,2 \mathrm{~g} / \mathrm{m}^{2} /$ año en verano con carga simple. Sin embargo, los valores ascendieron a $90,3 \mathrm{~g} / \mathrm{m}^{2} /$ año y $36,5 \mathrm{~g} / \mathrm{m}^{2} /$ año con carga doble, respectivamente. En la Tabla 2 se incluye la proporción consumida con ambas cargas respecto a la producción.

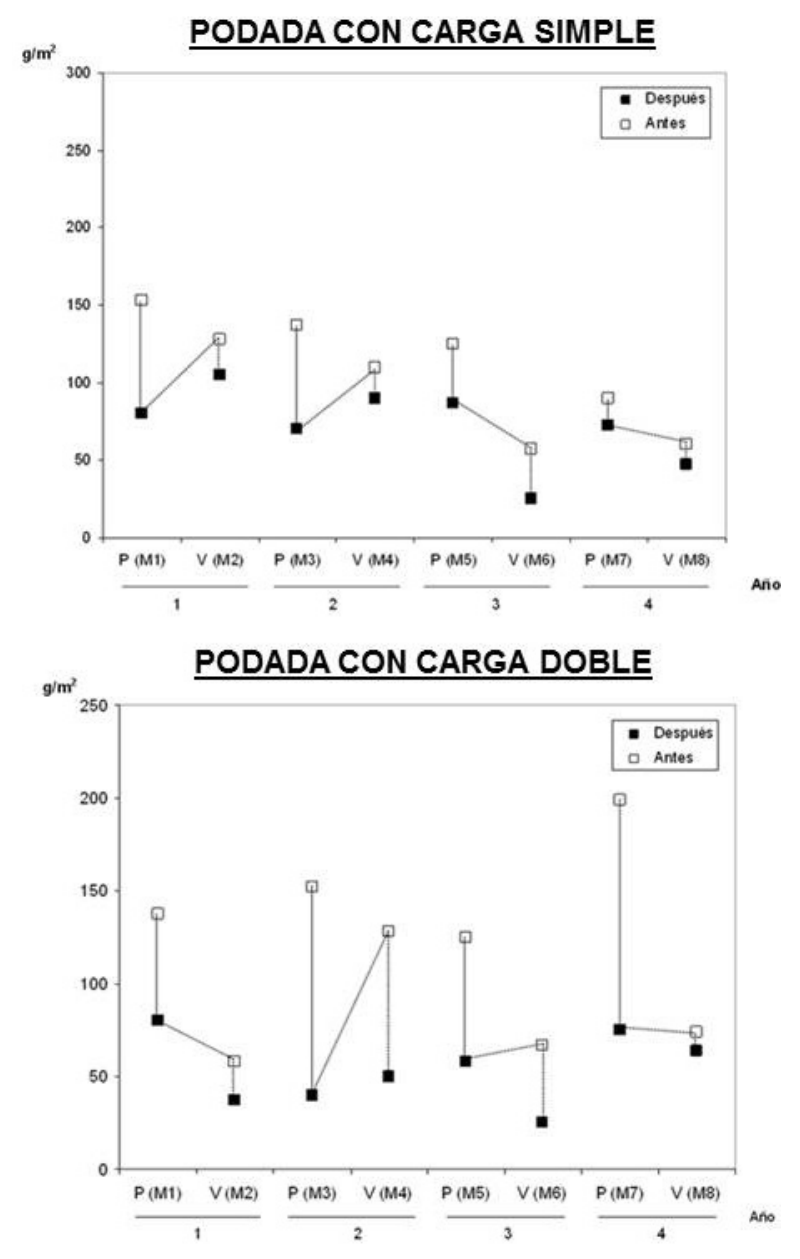

Figura 2: Evolución de la producción herbácea $\left(\mathrm{g} / \mathrm{m}^{2}\right)$ en los ocho pastoreos. P: primavera; V: verano; M: muestreo.

Figure 2: Evolution of the herbaceous production $\left(\mathrm{g} / \mathrm{m}^{2}\right)$ in the eight grazing areas. P: spring; V: summer; M: sampling. 
Tabla 2: Valores medios de producción de herbáceas y de Cytisus scoparius $\left(\mathrm{g} / \mathrm{m}^{2} \mathrm{MS}\right)$ en primavera y verano, con su error estándar, y consumo (\%).

Table 2: Mean average herbaceous and Cytisus scoparius production $\left(\mathrm{g} / \mathrm{m}^{2} \mathrm{MS}\right)$ in spring and summer, including the standard error, and consumption (\%).

\begin{tabular}{|c|c|c|c|}
\hline \multirow[b]{2}{*}{ Carga simple } & \multicolumn{2}{|c|}{ Producción } & \multirow{2}{*}{$\begin{array}{c}\text { Consumo } \\
(\%)\end{array}$} \\
\hline & $\mathrm{g} / \mathrm{m}^{2} \mathrm{MS}$ & $\begin{array}{c}\text { Error } \\
\text { estándar }\end{array}$ & \\
\hline $\begin{array}{l}\text { Herbáceas en } \\
\text { primavera }\end{array}$ & 132,6 & 14,4 & 36,6 \\
\hline $\begin{array}{l}\text { Herbáceas en } \\
\text { verano }\end{array}$ & 94,4 & 17,9 & 22,5 \\
\hline $\begin{array}{l}\text { Cytisus en } \\
\text { primavera }\end{array}$ & 86,8 & 40,1 & 43,8 \\
\hline Cytisus en verano & 196,3 & 24,8 & 36,2 \\
\hline \multicolumn{4}{|l|}{ Carga doble } \\
\hline $\begin{array}{l}\text { Herbáceas en } \\
\text { primavera }\end{array}$ & 160 & 15,5 & 56,4 \\
\hline $\begin{array}{l}\text { Herbáceas en } \\
\text { verano }\end{array}$ & 88,5 & 16,0 & 41,2 \\
\hline $\begin{array}{l}\text { Cytisus en } \\
\text { primavera }\end{array}$ & 36,3 & 17,7 & 63 \\
\hline Cytisus en verano & 45,4 & 1,5 & 70,8 \\
\hline
\end{tabular}

\subsection{Análisis del paisaje}

La Figura 3 incluye la evolución de la cubierta de Cytisus scoparius antes del primer pastoreo de cada año, es decir los pastoreos $1,3,5$ y 7 , tanto en la subparcela sometida a carga simple (Figura 3A) como en la sometida a carga doble (Figura 3B). La mera observación de la Figura 3

\section{A: SUBPARCELA PASTADA CON CARGA SIMPLE}

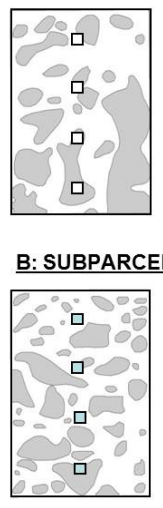

M1
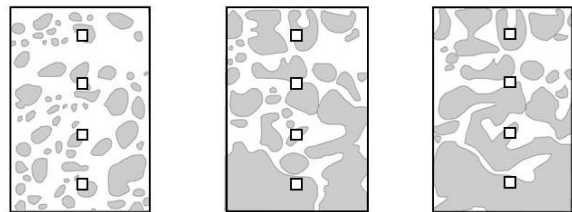

$\square$

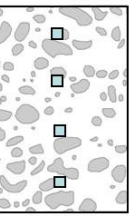

M7
Figura 3: Evolución de la cubierta de Cytisus scoparius.

A: Subparcela pastada con carga simple. B: Subparcela pastada con carga doble.

Figure 3: Evolution of Cytisus scoparius coverage. A: Subplot grazed with single rate. B: Subplot grazed doubly rate.

pone de relieve la mayor fragmentación de la subparcela pastada con carga doble que la pastada con carga simple; en esta última las manchas se van fusionando hasta que la subparcela queda cubierta por muy pocas manchas.

Los resultados obtenidos al aplicar métricas espaciales (Ver Tabla 3) confirman la observación visual (Fotos 3 y 4). Después de la poda, el número de manchas de matorral aumenta, pasando entre el primer y tercer pastoreo de 21 manchas a 65 (incremento del 309,5\%) con carga simple y de 48 manchas a 88 (incremento del 183,3\%) con carga doble. La evolución a partir de ese momento es diferente entre ambos sistemas de gestión: con carga simple se reduce

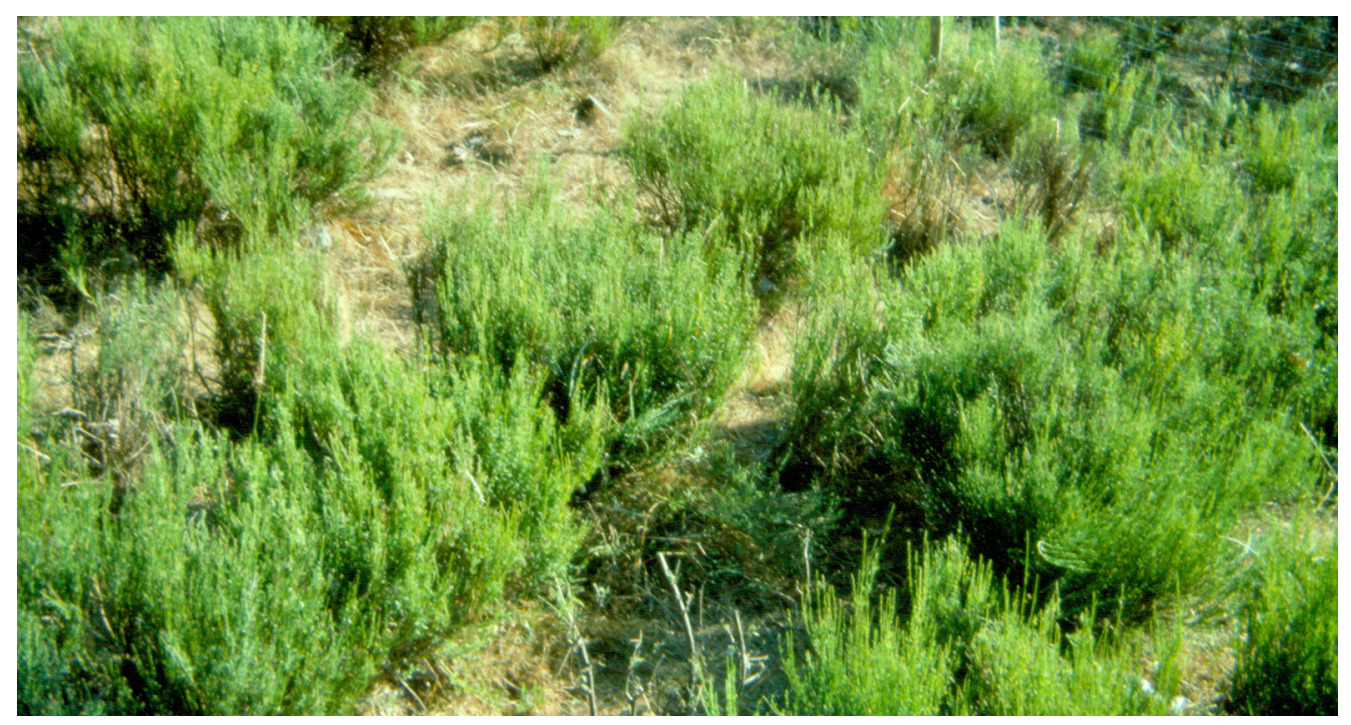

Foto 3. Subparcela pastada con carga simple.

Photo 3. Subplot grazed with single grazing rate. 


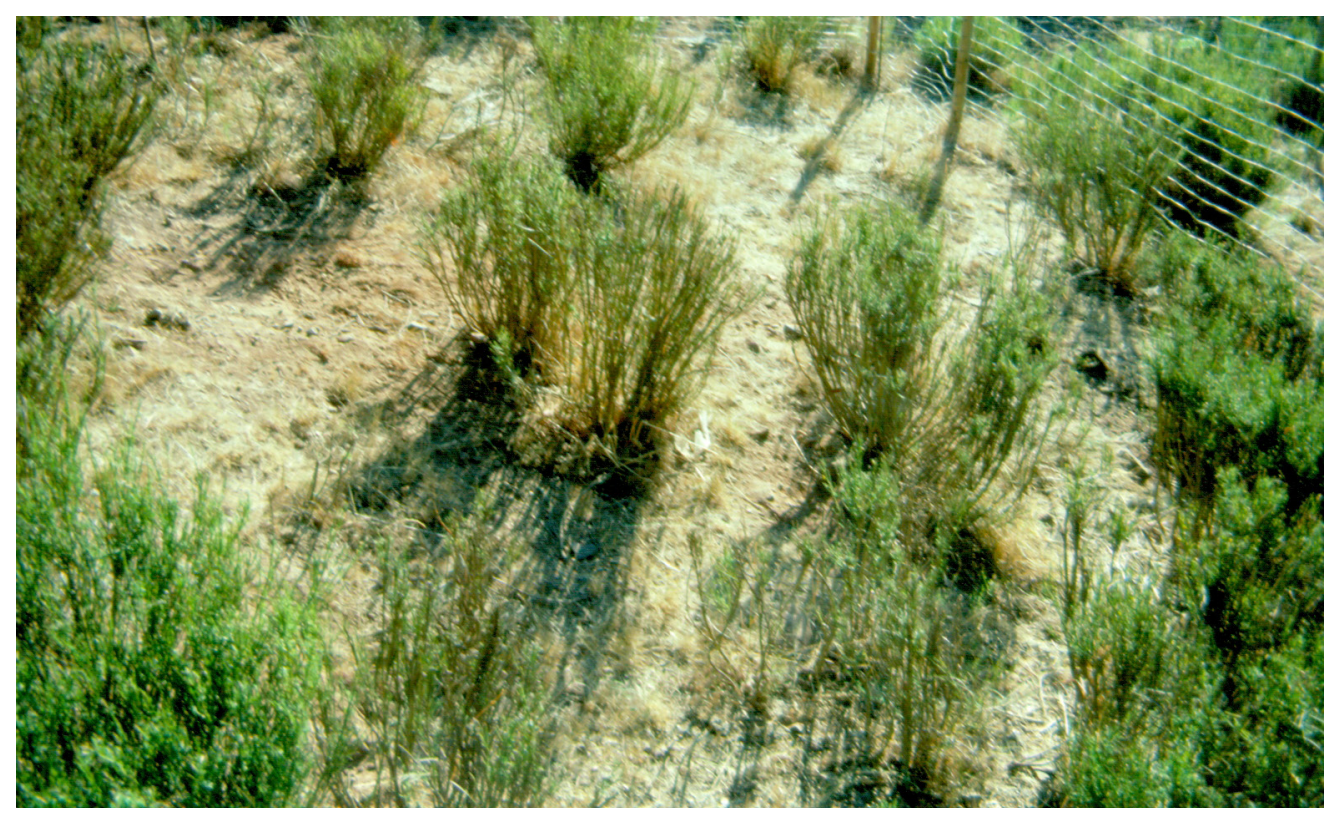

Foto 4: Subparcela pastada con carga doble.

Photo 4: Subplot grazed with double grazing rate.

el número de manchas (18 en el pastoreo 7) por unión de unas manchas con otras, mientras que con carga doble se mantiene el número de manchas ( 85 en el pastoreo 7 ).

En el inicio del experimento (Ver Figura 3) podemos considerar que la superficie total de las manchas es muy parecida, ya que ocupaban $42,2 \mathrm{~m}^{2}$ en la parcela A y 35,4 $\mathrm{m}^{2}$ en la parcela B. Estos datos implican que la superficie ocupada por las herbáceas era de $53,8 \mathrm{~m}^{2}$ y $60,6 \mathrm{~m}^{2}$, respectivamente. Durante el primer año la superficie de las manchas de matorral decrece en ambos sistemas de gestión, para a continuación aumentar con carga simple (61,5 $\mathrm{m}^{2}$ en el pastoreo 7) y descender ligeramente con carga doble $\left(26 \mathrm{~m}^{2}\right.$ en el pastoreo 7). El tamaño medio de las manchas guarda relación, lógicamente, con la superficie y número de las mismas, siendo menor con carga doble. La longitud de los ejes es ligeramente superior en la parcela gestionada con carga doble en relación con el mayor número de manchas, mientras que el tamaño medio de los ejes es inferior, al ser más pequeñas las manchas.

El índice medio de formas (IMF) no muestra grandes diferencias entre ambos sistemas de gestión, poniendo de relieve en ambos casos que las manchas se aproximan más a un círculo que a una línea recta. No obstante, merece la pena destacar que con carga simple y durante el pastoreo 7 se obtiene el valor más alto $(1,326)$, dando a entender que la unión entre manchas tiende a formar polígonos alargados (Ver Figura 3A). Por el contrario, en la carga doble el valor del IMF tiende a disminuir, generándose manchas progresivamente más circulares (Ver Figura $3 \mathrm{~B})$. La relación perímetro - área da valores más bajos

Tabla 3: Evolución de varios parámetros del paisaje en función de la carga de pastoreo.

Table 3: Evolution of several landscape parameters related to grazing rates.

\begin{tabular}{|c|c|c|c|c|c|c|c|c|c|}
\hline & \multicolumn{4}{|c|}{ Carga simple } & \multicolumn{4}{|c|}{ Carga doble } \\
\hline & & Pastoreo 1 & Pastoreo 3 & Pastoreo 5 & Pastoreo 7 & Pastoreo 1 & Pastoreo 3 & Pastoreo 5 & Pastoreo 7 \\
\hline \multirow{4}{*}{ Área } & N. ${ }^{\circ}$ de manchas & 21 & 65 & 26 & 18 & 48 & 88 & 86 & 85 \\
\hline & Superficie de las manchas $\left(\mathrm{m}^{2}\right)$ & 42,19 & 28,32 & 59,35 & 61,5 & 35,39 & 23,58 & 34,97 & 26,03 \\
\hline & Tamaño medio de la mancha & 2,01 & 0,44 & 2,28 & 3,42 & 0,74 & 0,27 & 0,41 & 0,31 \\
\hline & Densidad de manchas & 3,37 & 0,55 & 3,98 & 8,84 & 0,93 & 0,28 & 0,41 & 0,32 \\
\hline \multirow{2}{*}{ Bordes } & Longitud (m) & 111,7 & 145,4 & 141,9 & 137,3 & 145,9 & 166 & 200 & 164 \\
\hline & Media de longitud & 5,32 & 2,24 & 5,46 & 7,63 & 3,04 & 1,89 & 2,33 & 1,93 \\
\hline \multirow{3}{*}{ Formas } & Índice Medio de Formas (IMF) & 1,196 & 1,096 & 1,194 & 1,326 & 1,138 & 1,125 & 1,147 & 1,113 \\
\hline & Relación perímetro - área & 4,832 & 9,012 & 5,873 & 6,375 & 7,39 & 10,38 & 9,587 & 11,343 \\
\hline & Dimensión fractal (F) & 1,317 & 1,356 & 1,326 & 1,346 & 1,343 & 1,375 & 1,368 & 1,380 \\
\hline
\end{tabular}


para cada pastoreo en la parcela con carga doble. La dimensión fractal muestra el comportamiento inverso, si bien los valores son muy parecidos entre ambas cargas de pastoreo. Tales valores oscilan entre 1,31 y 1,38 , es decir que se acercan más a un polígono regular que de grandes sinuosidades.

\section{Discusión}

La ganadería extensiva constituye un elemento esencial para la gestión del territorio y la configuración del paisaje (Gibon, 2005). A través de la deforestación, quema, desbroce y poda el hombre ha obtenido pastos para la ganadería a la vez que causaba destacados efectos sobre el territorio: Lasanta (2010) incluye un buen número de citas bibliográficas relacionadas con estos temas. En muchas regiones del Mundo se sigue deforestando y quemando todavía para ampliar la extensión de los pastos (Genin et al., 1994).

En las montañas de los países desarrollados, por el contrario, se asiste a un intenso proceso de revegetación, con avance -con frecuencia indiscriminado- de matorrales y bosques como consecuencia de la marginación del sector primario, lo que tiene implicaciones en el incremento del riesgo de incendios, en el ciclo hidrológico, en la conservación del suelo, en la disponibilidad de pastos, en la biodiversidad, y en la diversidad y calidad estética del paisaje (Antrop, 1993; Lasanta et al., 2006). Con el fin de regenerar pastos, y a la vez controlar los incendios y mantener la heterogeneidad del paisaje, se recurre a la ganadería extensiva (Bernués, 2007). Sin embargo, el ganado por sí sólo se muestra incapaz para transformar áreas extensas de matorral en pastos (Bartolomé et al., 2000). Heineman et al. (2005) comprobaron que ni el desbroce químico, ni el manual, ni el pastoreo eran prácticas suficientes para mantener los pastos, si las intervenciones anteriores se realizaban independientemente.

En este trabajo se ha tratado de dar un paso más: Se ha llevado a cabo un ensayo de campo combinando la poda de Cytisus scoparius con el pastoreo de cabras, empleando dos cargas ganaderas: simple (4,5 cabras/ha/año) y doble ( 9 cabras/ha/año). Con este trabajo inicial tan sólo se quiere aportar unos primeros resultados, que se deberían confirmar en estudios posteriores con mayor número de parcelas. No obstante, la información obtenida resulta muy interesante y esperanzadora; apunta a que la poda por sí sola tiene escasa capacidad para limitar la expansión de los matorrales, ya que la superficie con cubierta arbustiva vuelve a cubrir el $100 \%$ durante el tercer año y el $116 \%$ el cuarto año, al entremezclarse matas de Cytisus scoparius y Rubus idaeus. Por otro lado, cuatro años después de la poda las ramas de Cytisus scoparius recuperan el $85 \%$ de la altitud previa a la poda.

Por el contrario, la combinación poda - pastoreo parece ejercer, con las dos cargas estudiadas en este trabajo, cierto efecto en el control de las matas de Cytisus scoparius. Con carga simple el efecto es bastante limitado, ya que el consumo medio de los brotes representa el
$40 \%$ de lo ofertado (113,2 $\mathrm{g} / \mathrm{m}^{2}$ de MS), lo que implica que Cytisus scoparius se recupere paulatinamente; al cuarto año su altura alcanza el 70,6\% de la que tenía antes del desbroce, a la vez que su superficie pasa de tener $42,2 \mathrm{~m}^{2}$ durante el primer año a $61,5 \mathrm{~m}^{2}$ durante el cuarto año de experimentación. Si el pastoreo es con carga doble el consumo se eleva al 66,9\% de lo producido. En concreto, durante el primer año la superficie de los matorrales es de $35,4 \mathrm{~m}^{2}$, mientras que al cuarto año se ha reducido ligeramente, ocupando $26 \mathrm{~m}^{2}$. El consumo de brotes del año implica también una ralentización en la recuperación de la altura original de las matas, de forma que al cuarto año las ramas sólo miden $80 \mathrm{~cm}$, es decir que sólo han recuperado $10 \mathrm{~cm}$ tras la poda, frente a los $50 \mathrm{~cm}$ de crecimiento con carga simple y los $74 \mathrm{~cm}$ en la parcela control.

La poda implica abrir espacios para el desarrollo de una cubierta herbácea, que se incorpora a la oferta de pastos. Los resultados obtenidos permiten cuantificar la oferta en $132,6 \mathrm{~g} / \mathrm{m}^{2}$ de MS en la parcela gestionada con carga simple y en $160 \mathrm{~g} / \mathrm{m}^{2}$ en la de carga doble. Un hecho a destacar es que la biomasa herbácea presenta una curva temporal descendente con carga simple y ascendente con carga doble, lo que -lógicamente- guarda una estrecha relación con la distinta evolución de los matorrales en ambas parcelas.

El uso de carga doble en la subparcela podada implica generar un paisaje configurado de pequeñas manchas de matorral, lo que resulta muy interesante desde una perspectiva ganadera, ya que ofrece varias ventajas: facilita el desarrollo de cubiertas herbáceas entre las matas, contribuyendo a la diversificación de la oferta pastoral y a mejorar la accesibilidad del ganado a las ramas. En este sentido, conviene recordar que los resultados obtenidos (ver Tabla 3) permiten concluir que la longitud de los bordes de las manchas es siempre mayor con carga doble que con carga simple. Sin embargo, el IMF no revela grandes diferencias entre las manchas de las dos parcelas, ya que en ambas se acercan más a manchas de forma circular que alargada, siendo las últimas mucho más interesantes, ya que incrementan la superficie expuesta a la boca del ganado (Burel y Baudry, 2002). La asociación entre herbáceas y matorrales resulta muy positiva al ofrecer recursos complementarios, tanto en la distribución estacional como en palatabilidad y componentes nutritivos (Montalvo et al., 1998). En nuestro estudio se ha podido ver la preferencia de la cabra por el consumo de brotes tiernos en verano, cuando las herbáceas se agostan, si puede seleccionar su dieta. Por otro lado, los arbustos proporcionan protección a las plantas de la radiación solar y a los animales de las inclemencias del tiempo (Narjisse, 1990). Asimismo, las raíces bombean nutrientes desde capas relativamente profundas del suelo hacia la superficie, mejorando la fertilidad y la oferta del pasto de herbáceas (Montserrat, 2008).

Un hecho muy destacable, desde una perspectiva paisajística, es el mayor grado de fragmentación del paisaje con carga doble que con carga simple, como lo demuestra el número de manchas y su menor tamaño. La fragmenta- 
ción tiene importantes implicaciones ambientales, al modificar la heterogeneidad interna de los hábitats (Freemark y Merriam, 1986). En áreas con dilatada historia de ocupación humana, como es nuestro caso, la fauna suele estar adaptada a paisajes heterogéneos, donde se combinan espacios abiertos (campos agrícolas, pastos,..) con zonas más cerradas (bosques, matorrales,..), lo que les permite tener áreas de alimentación y refugio. La disminución de la fragmentación favorece la desaparición de tales especies que son sustituidas por otras que requieren manchas muy extensas. Por otro lado, la permanencia de pequeñas manchas de matorral próximas genera corredores que facilitan la conectividad entre espacios (Burel y Baudry, 2002).

El control de los matorrales por la ganadería extensiva tiene otros efectos ambientales y socieconómicos, en la medida en que contribuye a eliminar combustible, e indirectamente a disminuir el riesgo de incendio, a diversificar el paisaje y a darle un mayor valor social (Magadlela et al., 1995; Torrano y Valderrábano, 2005; Baur et al., 2006; Casasús et al., 2007; Gibón et al., 2010) y perpetuar rasgos de un paisaje humanizado de alto interés cultural, que en parte ha perdido su valor, siendo necesario acudir a intervenciones programadas para mantenerlo. En relación con ello, Vanslemsbrouk et al. (2005) comprobaron que rasgos del paisaje asociados a actividades agrarias, en particular los prados y la ganadería extensiva, influyen de manera positiva en la demanda de turismo rural y en el precio que los turistas están dispuestos a pagar por su estancia vacacional.

\section{Conclusiones}

La despoblación de las áreas de montaña españolas y la disminución del ganado, que tradicionalmente consumía el pasto, han llevado a la expansión descontrolada de los matorrales y, consecuentemente, a un incremento significativo de material combustible y del riesgo de incendio, así como a la pérdida de heterogeneidad del paisaje. En la montaña Cantábrica, Cytisus scoparius constituye una especie con gran capacidad invasora, por lo que cubre elevadas extensiones de antiguas áreas de pastoreo.

Los resultados obtenidos en este trabajo apuntan a que ni la poda de Cytisus scoparius ni el pastoreo de matorrales con las actuales cargas ganaderas permiten controlar la expansión de los matorrales. Sin embargo, ambas prácticas conjuntas resultan más eficaces. Especialmente interesante resulta la carga doble ( 9 cabras/ha/año), ya que permite alimentar un censo mayor y mantener un paisaje fragmentado y más próximo a la estructura en mosaico de los paisajes tradicionales, de destacado valor cultural.

La conclusión a la que puede llegarse es que los paisajes creados con fuerte inversión de trabajo y energía, como los de las montañas con elevada carga histórica, siguen necesitando de inversiones para su mantenimiento. Se confirma también, una vez más, que la ganadería es un elemento de gestión imprescindible en los paisajes de nuestras montañas.

\section{Agradecimientos}

Este trabajo se ha realizado con el apoyo de los proyectos: INDICA (CGL2011-27753-CO2-01 y 02) e HIDROCAES (CGL2011-27574-CO2-02), financiados por el Ministerio de Economía y Competitividad (Gobierno de España). Ha contado también con la ayuda de la DGA al Grupo de Investigación: Geomorfología y Cambio Global.

\section{Referencias}

Álvarez-Martínez, J., 1995. Dinámica sucesional tras el abandono y recuperación del matorral mediante pastoreo controlado. Experiencia en un sector de la montaña de León. Universitat de Lleida, Servei de Publicacions. 357 pp., Lleida.

Antrop, M., 1993. The transformation of the Mediterranean landscapes: an experience of 25 years of observations. Landscape and Urban Planning, 24: 3-13. http://dx.doi. org/10.1016/0169-2046(93)90076-P

Arnaez, J., Lasanta, T., Errea, M.P. \& Ortigosa, L.M., 2011. Land abandonment, landscape evolution, and soil erosion in a Spanish Mediterranean. Land Degradation and Development, 22:537-550. http://dx.doi.org/10.1002/ldr.1032

Bartolomé, J. Franch, J., Plaixats, J. \& Seligman, N.G., 2000. Grazing alone is not enough to maintain landscape diversity in the Montseny Biosphere Reserve. Agriculture, Ecosystems and Environment, 77: 267-273. http://dx.doi.org/10.1016/ S0167-8809(99)00086-9

Bartolomé, J., Plaixats, J., Piedrafita, J., Fina, M., Adrobau, E., Aixàs, A., Bonet, M. Grau, J. \& Polo, L., 2011. Foraging behavior of Alberes cattle in a Mediterranean forest ecosystem. Rangeland Ecology Management, 64: 319-324. http:// dx.doi.org/10.2111/REM-D-09-00160.1

Baur, B., Cremene, C., Groza, G., Rakosy, L., Schileyko, A.A., Baur, A., Stoll, P. \& Erhardt, A., 2006. Effects of abandonment of subalpine hay meadows on plant invertebrate diversity in Transylvania, Romania. Biological Conservation, 132: 262-273. http://dx.doi.org/10.1016/j.biocon.2006.04.018

Bernués, A., 2007. Ganadería de montaña en un contexto global. Evolución, condicionantes y oportunidades. Pastos, 37 (2): 133-175.

Bielsa, I., Pons, X. \& Bunce, B., 2005. Agricultural abandonment in the North Eastern Iberian Peninsula: The use of basic landscape metrics to support planning. Journal of Environmental Planning and Management, 48(1): 85-102. http:// dx.doi.org/10.1080/0964056042000308166

Burel, F. \& Baudry, J., 2002. Ecología del paisaje. Conceptos, métodos y aplicaciones. Ediciones Mundi-Prensa: $353 \mathrm{pp}$. Madrid.

Casasús, I., Bernués, A., Sanz, A., Villalba, D., Riedel, J.L. \& Revilla, R., 2007. Vegetation dynamics in Mediterranean forest pastures as affected by beef cattle grazing. Agriculture, Ecosystems and Environment, 121: 365-370. http://dx.doi. org/10.1016/j.agee.2006.11.012

Cascos Maraña, C. 2011. La simplificación y la uniformidad crecientes en los paisajes ganaderos de la montaña húmeda de Castilla y León. El ejemplo de Babia y Luna. Polígonos. Revista de Geografia, 21: 149-178.

Collantes, F. G., 2004. El declive demográfico de la montaña española (1850-2000) ¿Un drama rural? Ministerio de Agricultura, Pesca y Alimentación. Secretaria General Técnica. Serie Estudios, 159: 364 pp., Madrid. 
Errea, M.P., Arnáez, J., Ortigosa, L., Oserin, M., Ruiz-Flaño, P. \& Lasanta, T., 2007. Marginación y paisaje en una montaña submediterránea (1956-2011): El ejemplo de Camero Viejo (Sistema Ibérico, La Rioja). Nimbus, 19-20: 53-71.

Farina, A., 2007. Principles and methods of landscape ecology towards a science of the landscape. Springer-Verlag, 2nd ed., New York, NY.

Forman, R.T.T. \& Godron, M., 1986. Landscape Ecology. John Wiley \& Sons, New York

Freemark, K.E. \& Merrian, H.G., 1986. Importance of area and habitat heterogeneity to bird assemblages in temperature forest fragments. Biological Conservation, 36: 115-141. http://dx.doi.org/10.1016/0006-3207(86)90002-9

García-Ruiz, J.M. \& López Bermúdez, F., 2009. La erosión del suelo en España. Sociedad Española de Geomorfología: 441 pp., Zaragoza.

García-Ruiz, J.M., Lana-Renault, N., Beguería, S., Lasanta, T., Regüés, D., Nadal-Romero, E., Serrano-Muela, P., LópezMoreno, J.I., Alvera, B., Martí-Bono, C. \& Alatorre, L.C., 2010. From plot to regional scales: Interactions of slope and catchment hydrological and geomorphic processes in the Spanish Pyrenees. Geomorphology, 120 (3-4): 248-257. http://dx.doi.org/10.1016/j.geomorph.2010.03.038

Gellrich, M., Baur, P., Koch, B. \& Zimmermann, N.E., 2007. Agricultural land abandonment and natural forest re-growth in the Swiss mountains: A spatially explicit economic analysis. Agriculture, Ecosystems and Environment, 118: 93-108. http://dx.doi.org/10.1016/j.geomorph.2010.03.038

Genin, D., Villca, Z. \& Abasto, P., 1994. Diet selection and utilization by llama and sheep in high altitude arid rangeland of Bolivia. Journal of Range Management, 47: 245-248. http:// dx.doi.org/10.2307/4003025

Gibon, A., 2005. Managing grassland for production, the environment and the landscape. Chalenges at the farm and the landscape level. Livestock Production Science, 96: 11-31. $\mathrm{http}: / / \mathrm{dx}$.doi.org/10.1016/j.livprodsci.2005.05.009

Gibon, A., Sheeren, D., Monteil, C., Ladet, S. \& Balent, G., 2010. Modelling and simulating change in reforesting mountain landscapes using a social-ecological framework. Landscape Ecology, 25: 267-285. http://dx.doi.org/10.1007/ s10980-009-9438-5

Glasser, T.A., Landau, S.Y., Ungar, E.D., Perevolotsky, A., Dvash, L., Muklada, H., Kababya, D. \& Walker, J.W., 2012. Foraging selectivity of three goat breeds in a Mediterranean shrubland. Small Ruminant Research, 102: 7-12. http:// dx.doi.org/10.1016/j.smallrumres.2011.09.009

Gómez García, D. Aguirre, A.J. \& Lizaur Sukia, X., 2011. Recuperación del matorral tras el desbroce mecánico y quema en pastos de la Sierra de Aralar y Belate (Navarra). En: López, C., Rodríguez, M.P., San Miguel, A., Fernández, F. y Roig, S. Pastos, paisajes culturales entre tradición y nuevos paradigmas del siglo XXI. Sociedad Española para el Estudio de los Pastos, 133-138 pp., Toledo.

Hart, S.P., 2001. Recent perspectives in using goats for vegetation management in the USA. Journal of Dairy Science, 74: 170176. http://dx.doi.org/10.3168/jds.S0022-0302(01)70212-3

Heineman, J.L., Simard, S.W., Sachs, D.L. \& Mather, W.J., 2005. Chemical, grazing, and manual cutting treatments in mixed herb-shrub communities have no effect on interior spruce survival or growth in southern interior British Columbia. Forest Ecology and Management, 205: 359-374. http://dx.doi.org/10.1016/j.foreco.2004.10.038

Hofmann, R.R., 1989. Evolutionary steps of ecophysiological adaptation and diversification of ruminants: comparative view of their digestive system. Oecologia, 78: 443-457. http://dx.doi.org/10.1007/BF00378733

Kienast, F., 1993. Analysis of historical landscape patterns with a Geographical Information System -a methodological outline.
Landscape Ecology, 8: 103-118. http://dx.doi.org/10.1007/ BF00141590

Koutsias, N., Martínez Fernández, J. \& Allgöwer, B., 2010. Do factors causing wildfires vary in space? Evidence from geographically weighted regression. GIScience and Remote Sensing, 47 (2): 221-240. http://dx.doi.org/10.2747/1548-1603.47.2.221

Lasanta, T., 1990. Tendances actuelles de l'organisation spatiale des montagnes espagnoles. Annales de Géographie, 551: 51-71. http://dx.doi.org/10.3406/geo.1990.20944

Lasanta, T., 2010. Pastoreo en áreas de montaña: Estrategias e impactos en el territorio. Estudios Geográficos, 268: 203233. http://dx.doi.org/10.3989/estgeogr.0459

Lasanta, T., Errea, M.P., Arnáez, J., Ortigosa, L. \& Ruiz-Flaño, P., 2009. Mountain pastures, environmental degradation, and landscape remediation: The example of a Mediterranean Policy Initiative. Applied Geography, 29: 308-319. http://dx.doi.org/10.1016/j.apgeog.2008.09.006

Lasanta, T., González-Hidalgo, J.C., Vicente-Serrano, S.M. \& Sferi, E., 2006. Using landscape ecology to evaluate an alternative management scenario in abandoned Mediterranean mountainous areas. Landscape and Urban Planning, 78: 101114. http://dx.doi.org/10.1016/j.landurbplan.2005.06.003

Lasanta, T., Nadal-Romero, E., Errea, P. y Arnáez, J. 2015. The effects of landscape conservation measures in changing landscape patterns: A case study in Mediterranean mountains. Land Degradation \& Development. http://dx.doi.org/ 10.1002/ldr.2359.

Lasanta, T. \& Vicente-Serrano, S.M., 2007. Cambios en la cubierta vegetal en el Pirineo aragonés en los últimos 50 años. Pirineos, 162: 125-154.

Lasanta, T., Vicente-Serrano, S.M. \& Cuadrat, J.M., 2005. Mountain Mediterranean landscape evolution caused by the abandonment of traditional primary activities: a study of the Spanish Central Pyrenees. Applied Geography, 25: 47-65. http://dx.doi.org/10.1016/j.apgeog.2004.11.001

López-Moreno, J.I., Beguería, S., Valero, B. \& García-Ruiz, J.M., 2003. Intensidad de avenidas y aterramiento de embalses en el Pirineo Central español. Ería, 61: 159-167.

Lovejoy, S., 1982. Area-perimeter relation for rain and cloud areas. Science, 216: 185-187. http://dx.doi.org/10.1126/ science. 216.4542 .185

Magadlela, A.M., Dabaan, M.E., Bryan, W.B., Prigge, E.C., Skousen, J.G., D'Soura, G.E., Arbogast, B.L. \& Flores, G., 1995. Brush clearing on hill land pasture with sheep and goats. Journal of Agro Grop Science, 174: 1-8. http://dx.doi. org/10.1111/j.1439-037X.1995.tb00188.x

Mandelbrot, B.B., 1983. The fractal geometry of nature. W.H. Freeman and Company, 495 pp., New York.

McGarigal, K., Cushman, S.A., Neel, M.C. \& Ene, E., 2002. FRAGSTATS: Spatial pattern Analysis Program for categorical Maps. University of Massachusetts, Amherst.

Molinillo, M., Lasanta, T. García-Ruiz, J.M., 1997. Managing degraded landscape after farmland abandonment in the Central Spanish Pyrenees. Environmental Management, 21: 587-598. http://dx.doi.org/10.1007/s002679900051

Montalvo, J., De Miguel, J.M., Schmitz, M.F., García-Criado, B. \& Pineda, F.D., 1998. Calidad de la hierba y sucesión secundaria en un pastizal mediterráneo. Monografias del Instituto Pirenaico de Ecología, 4: 837-846.

Montserrat, P., 2008. Ecología del pasto pirenaico. Un panorama general. En: F. Fillat, R. García-González, D. Gómez \& R. Reiné, 2008. Eds. Pastos del Pirineo CSIC y Diputación de Huesca, 1-4 pp., Huesca.

Mottet, A., Ladet, S., Coque, N. \& Gibón, A., 2006. Agricultural land-use change and its drivers in mountain landscapes: A case study in the Pyrenees. Agriculture, Ecosystems and Environment, 114: 296-310. http://dx.doi.org/10.1016/j. agee.2005.11.017 
Narjisse, H., 1990. Les resources sylvo-pastorales en milieu méditerranéen. En: H. Narjisse \& A. Kabbli, Eds: L'élevage dans les systèmes cerealières méditerranéens Pudoc, Wageningen (Netherland), 353-393 pp.

Nogués-Bravo, D., 2006.Assessing the effects of environmental and anthropogenic factor on land-cover diversity in a Mediterranean mountain environment. Area, 38(4): 432-444. http://dx.doi.org/10.1111/j.1475-4762.2006.00709.x

Olsson, E.G.A., Austrheim, G. \& Grenne, S.N., 2000. Landscape change patterns in mountains, land use and environmental diversity, Mid-Norway 1960-1993. Landscape Ecology, 15: 155-170. http://dx.doi.org/10.1023/A:1008173628016

Ortuño, S.F. \& Martín Fernández, A.J., 2006. Forest externalities, demography and rural development in inland Spain. Forest Policy and Economics, 8: 109-122. http://dx.doi. org/10.1016/j.forpol.2004.05.007

Papadakis, J., 1966. Climates of the world and their agricultural potentialities. Buenos Aires.

Puentes Graña, M.A., 1984. Estrategias de regeneración del tojo tras el incendio. Memoria de Licenciatura. Universidad de Santiago de Compostela.

Pueyo, Y. \& Beguería, S., 2007. Modelling the rate of secondary succession after farmland abandonment in a Mediterranean mountain area. Landscape and Urban Planning, 83: 245254. http://dx.doi.org/10.1016/j.landurbplan.2007.04.008

Rescia, A.J., Pons, A., Lomba, I., Esteban, C. \& Dover, J.W., 2008. Reformulating the socio-ecological system in a cultural rural mountain landscape in the Picos de Europa region (Northern Spain). Landscape and Urban Planning, 28: 2333. http://dx.doi.org/10.1016/j.landurbplan.2008.08.001

Riedel, J.L., 2007. Bases para la gestión del Parque Natural de la Sierra y cañones de Guara: interacciones entre la ganadería y la dinámica de la vegetación. Tesis Doctoral. Universidad de Zaragoza: 215 pp., Zaragoza.

Rivas Martínez, S., Gandullo, J.M., Allúe, J.L., Montero de Burgos, J.L. \& González Rebollar, J.L., 1987. Memoria del mapa de series de vegetación de España. Ministerio de Agricultura, Pesca y Alimentación, Icona, Serie Técnica: 268 pp., Madrid.

Robert, M. \& Saugier, B., 2003. Contribution des écosystèmes continentaux à la séquestration du carbone. C.R. Geoscience, 335: 557-595. http://dx.doi.org/10.1016/S1631-0713(03)00094-4

Ruiz Mirazo, J., 2011. Environmental benefits of extensive livestock farming: wildfire prevention and beyond. Options Méditerranéennes, 100: 75- 82.
Ruiz Mirazo, J., Robles, A.B. \& González Rebollar, J.L., 2011. Two-year evaluation of fuelbreaks grazed by livestock in the wildfire prevention program in Andalusia (Spain). Agriculture, Ecosystems and Environment, 141: 13-22. http:// dx.doi.org/10.1016/j.agee.2011.02.002

Suárez-Seoane, S., 1998. Efectos ecológicos derivados del abandono de tierras de cultivo en la provincia de León (municipio de Chozas de Abajo). Tesis Doctoral. Universidad de León-INRA SAD Armorique: 281, Rennes.

Sayadi, S., González-Roa, M.C. \& Calatrava-Requena, J., 2009. Public preferences for landscape features: the case of agricultural landscape in mountainous Mediterranean areas. Land Use Policy, 26: 334-344. http://dx.doi.org/10.1016/j. landusepol.2008.04.003

Suárez-Seoane, S., Osborne, P.E. \& Baudry, J., 2002. Responses of birds of different biogeographic origins and habitat requirements land abandonment in Northern Spain. Biological Conservation, 105: 333-344. http://dx.doi.org/10.1016/ S0006-3207(01)00213-0

Tasser, E., Walde, J., Tappeiner, U., Teutsch, A. y Noggler, W., 2007. Land-use changes and natural reforestation in the Eastern Central Alps. Agriculture, Ecosystems and Environment, 118: 115-129. http://dx.doi.org/10.1016/j.agee.2006.05.004

Torrano, L. \& Valderrábano, J., 2005. Grazing ability of European black pine understory vegetation by goats. Small Ruminant Research, 58: 253-263. http://dx.doi.org/10.1016/j. smallrumres.2004.11.001

Trias, A., 1996. Control biológico del crecimiento del sotobosque con caprinos. Pastos, 26: 77-88.

Valderrábano, J. \& Torrano, L., 2000. The potential for using goats to control Genista scorpius shrubs in European black pines stands. Forest Ecology and Management, 126: 377383. http://dx.doi.org/10.1016/S0378-1127(99)00108-5

Vanslemsbrouck, I., Van Huylenbroeck, G. \& Van Meensel, J., 2005. Impact of agriculture on rural tourism: a hedonic pricing approach. Journal of Agricultural Economics, 56: 1730. http://dx.doi.org/10.1111/j.1477-9552.2005.tb00120.x

Vila Subirós, J., Ribas Palom, A., Varga Linde, D. \& Llausàs Pascual, A., 2009. Medio siglo de cambios paisajísticos en la montaña mediterránea. Percepción y valoración social del paisaje de la Alta Garrotxa. Pirineos, 164: 69-92. http:// dx.doi.org/10.3989/pirineos.2009.v164.30

Weng, Y.C., 2007. Spatio-temporal changes of landscape pattern in response to urbanization. Landscape and Urban Planning, 81: 341-353. http://dx.doi.org/10.1016/j.landurbplan.2007.01.009 